University of Texas Rio Grande Valley

ScholarWorks @ UTRGV

Health \& Human Performance Faculty

Publications and Presentations

\title{
Hemodynamic response and pulse wave analysis after upper- and lower-body resistance exercise with and without blood flow restriction
}

\author{
Yu Lun Tai \\ The University of Texas Rio Grande Valley \\ Erica M. Marshall \\ Jason C. Parks \\ J. Derek Kingsley
}

Follow this and additional works at: https://scholarworks.utrgv.edu/hhp_fac

Part of the Sports Sciences Commons

\section{Recommended Citation}

This is an Accepted Manuscript of an article published by Taylor \& Francis in European College of Sport Science on 16 September 2021, available online: http://www.tandfonline.com/10.1080/

17461391.2021.1982018

This Article is brought to you for free and open access by the College of Health Professions at ScholarWorks @ UTRGV. It has been accepted for inclusion in Health \& Human Performance Faculty Publications and Presentations by an authorized administrator of ScholarWorks @ UTRGV. For more information, please contact justin.white@utrgv.edu,william.flores01@utrgv.edu. 
Publisher: Taylor \& Francis \& European College of Sport Science

Journal: European Journal of Sport Science

DOI: 10.1080/17461391.2021.1982018

Hemodynamic response and pulse wave analysis after upper- and lower-body resistance exercise with and without blood flow restriction

Yu Lun Tai ${ }^{1,4}$, Erica M. Marshall ${ }^{2,4}$, Jason C. Parks ${ }^{3,4}$, J. Derek Kingsley ${ }^{4}$

${ }^{1}$ Department of Health and Human Performance, University of Texas Rio Grande Valley, Brownsville, TX, 78520, USA

${ }^{2}$ Florida Southern College, Lakeland, FL, 33801, USA

${ }^{3}$ State University of New York Cortland, Cortland, NY, 13045, USA

${ }^{4}$ Exercise Physiology, Kent State University, Kent, OH, 44242, USA

Corresponding author: Yu Lun Tai, email: yulun.tai@utrgv.edu
Abstract
Resistance exercise (RE) has been shown to elevate hemodynamics and pulse wave reflection. However, the effects of acute RE with blood flow restriction (BFR) on hemodynamics and pulse wave reflection are unclear. The purpose of this study was to evaluate the differences between upper- and lower-body RE with and without BFR on hemodynamics and pulse wave reflection. Twenty-three young resistance-trained individuals volunteered for the study. Hemodynamics and pulse wave reflection were assessed at rest, 10, 25, 40, and 55 minutes after either upper- or lower-body with or without BFR. The upper-body RE (URE) consisted of the latissimus dorsi pulldown and chest press; the lower-body RE (LRE) consisted of knee extension and knee 
flexion. The BFR condition consisted of four sets of 30,15, 15, and 15 repetitions at 30\% 1repetition maximum (1RM) while the without BFR condition consisted of four sets of 8 repetitions at 70\% 1RM. Heart rate, rate pressure product, and subendocardial viability ratio significantly $(\mathrm{p}<0.05)$ increased after all exercises. Brachial and aortic systolic blood pressure (BP) significantly $(\mathrm{p}<0.05)$ elevated after LRE while brachial and aortic diastolic BP significantly $(\mathrm{p}<0.05)$ reduced after URE. Augmentation pressure, augmentation index (AIx), AIx normalized at $75 \mathrm{bpm}$, and wasted left ventricular pressure energy significantly $(\mathrm{p}<0.05)$ increased after URE while transit time of reflected wave significantly $(\mathrm{p}<0.05)$ decreased after LRE. URE places greater stress on pulse wave reflection while LRE results in greater responses in BP. Regardless of URE or LRE, the cardiovascular responses between BFR and without BFR are similar.

\section{Highlights}

- High-load resistance exercise and low-load resistance exercise with blood flow restriction may produce similar cardiovascular responses.

- Upper-body resistance exercise generates greater changes on pulse wave reflections while lower-body resistance exercise induces greater elevations in systolic blood pressure.

Keywords: Arterial stiffness, augmentation index, blood pressure, pulse wave reflection

\section{INTRODUCTION}

The American College of Sports Medicine recommends resistance exercise (RE) for individuals to increase muscular strength and mass. ${ }^{1}$ In order to improve muscular properties, the recommended load is $\sim 50 \%$ of 1-repetition maximum (1RM) for beginners, while advanced lifters require greater loads (>80\% 1RM) to mediate further adaptations and strength gains. ${ }^{1}$ Based on a meta-analysis by Lixandrão et al. (2018), in order to combat these high loads, the application of blood flow restriction (BFR) with RE has been reported to increase muscular strength and mass at relatively low loads $(20-40 \% 1 \mathrm{RM}){ }^{2}$ This reduction in workload with BFR 
may reduce the effects of the workload on cardiovascular parameters; however, the data are inconclusive. ${ }^{3,4}$

Blood pressure (BP) is an important maker to assess the risk of cardiovascular disease. ${ }^{5}$ However, the hemodynamics after upper-body RE with or without BFR are mixed. Numerous studies have demonstrated that upper-body RE without BFR significantly increased brachial systolic BP (BSBP) and decreased brachial diastolic BP (BDBP). ${ }^{6-8}$ However, other studies have reported no changes in BSBP or BDBP after upper-body RE with ${ }^{4,9}$ or without $\mathrm{BFR}^{4,9}, 10$ Therefore, gaining more knowledge to better understand hemodynamics to upper-body RE with and without BFR is necessary.

The hemodynamics after lower-body RE with or without BFR are also inconsistent. Studies have shown no change in BSBP or BDBP after lower-body RE with ${ }^{11}{ }^{13}$ or without $\mathrm{BFR}^{11,13}$; therefore, the results are not constant. Some studies have reported that lower-body RE with $^{14}$ or without BFR ${ }^{14-16}$ significantly altered BSBP and BDBP. Figueroa and Vicil $(2011)^{14}$ reported that BSBP and BDBP increased immediately after lower-body RE with or without BFR while Heffernan et al. (2006 \& 2007) observed an increased BSBP at 5 minutes ${ }^{16}$ and a decreased BSBP at 20 minutes ${ }^{15}$ post-exercise. Collectively, the confounding results make it difficult to understand the responses and clearly more data are needed.

Pulse wave reflection, primarily augmentation pressure (AP), augmentation index (AIx), and AIx normalized at a heart rate (HR) of 75 bpm (AIx@75) are independent markers for cardiovascular diseases and all-cause mortality. ${ }^{17,18}$ The AP is the measure of additional pressure mediated by the backward traveling wave to the left ventricle ${ }^{19}$, while the AIx is the ratio of AP to pulse pressure (PP). ${ }^{20}$ In addition, an increase in AP and AIx results in increased left ventricle afterload and wasted left ventricle pressure energy $\left(\Delta \mathrm{E}_{\mathrm{w}}\right)$ which might place additional workload and increase myocardial oxygen demand on the left ventricle. ${ }^{21}$ Rate pressure product (RPP) is indicative of myocardial oxygen demand and subendocardial viability ratio (SEVR) is a reliable measure of myocardial perfusion, and is associated with microvascular function. ${ }^{22}$ The transit time of the reflected wave (Tr) is inversely associated with pulse wave reflection and arterial stiffness, and positively related to the length to the reflecting sites. ${ }^{23}$ The measures of pulse wave reflection provide information related to reservoir pressure and left ventricular function.

A previous study ${ }^{4}$ demonstrated that upper-body RE with or without BFR increased pulse wave reflection (AP, AIx, AIx@75, and $\Delta \mathrm{E}_{\mathrm{w}}$ ). This is in agreement with other studies in that 
upper-body RE with or without BFR elevated $\mathrm{AP}^{6}$, $\mathrm{AIx}^{6,8}$, and AIx @ 75. ${ }^{6-8}$ In contrast, previous findings have shown that lower-body $\mathrm{RE}$ with ${ }^{12,14}$ or without $\mathrm{BFR}^{14}$ decreased pulse wave reflection. Collectively, the data suggest that upper- versus lower-body RE with or without BFR may have different cardiovascular responses. However, to our knowledge, no study has evaluated upper- versus lower-body RE with BFR on cardiovascular parameters. In other words, the present study should provide practical applications for personal trainers and strength and conditioning coaches to better understand how to prescribe resistance exercise protocols.

Therefore, the purpose of present study was to evaluate the differences between upperand lower-body RE with and without BFR on hemodynamics and pulse wave reflection in young resistance-trained individuals. We hypothesized that hemodynamics would not change except HR, between upper- and lower-body RE with and without BFR, we also hypothesized that pulse wave reflection would be elevated except Tr and SEVR after upper-body RE with and without BFR compared to lower-body RE with and without BFR.

\section{METHODS}

\section{Subjects.}

Twenty-three resistance-trained individuals (14 men and 9 women) volunteered for the study. Our questionnaire sought to determine if our participants had been taking part in regular resistance training for $\geq$ three days/week for at least one year. Women completed all testing during the follicular phase of their menstrual cycles. Participants were excluded if they had smoking history ( $<6$ months), hypertension $(\geq 140 / 90 \mathrm{mmHg}$ ), obesity (body mass index $\geq 30$ $\mathrm{kg} / \mathrm{m}^{2}$ ), cardiovascular or metabolic diseases as assessed via Physical Activity Readiness Questionnaire and Health Participant Questionnaire. This research was approved by the Institutional Review Board and was completed in accordance with the Declaration of Helsinki.

\section{Procedures.}

This study used a counterbalanced within-subjects design in which participants came to the laboratory six times. The first visit consisted of assessments of anthropometric measurements, arterial occlusion pressure (AOP) of the right arm and right leg, and muscular strength. After $\geq$ 48 hours, the second visit consisted of assessment of AOP and verification of muscular strength. The following four testing days were separated by $\geq 72$ hours, participants were asked to avoid 
food for 3 hours, caffeine, alcohol, and strenuous exercise for 24 hours prior to testing. On testing days, participants reported to the laboratory, and data collection was completed after 10minute rest in the supine position. Participants performed either upper- or lower-body RE with or without BFR in a counterbalanced design then participants returned to the supine position with repeated the data collection at 10,25, 40, and 55 minutes post-exercise. All data collection was completed at the same time of the day ( \pm 1 hour), across the four testing days.

\section{Anthropometric measurement.}

Height and bodyweight were measured using a stadiometer and beam scale (Detecto 448 , Cardinal Scale Manufacturing, USA), respectively. Height was measured to the nearest $0.5 \mathrm{~cm}$ and converted to $\mathrm{m}$. Bodyweight was measured to the nearest $11 \mathrm{~b}$ and converted to $\mathrm{kg}$. Body Mass Index was calculated as bodyweight $(\mathrm{kg}) /$ height squared $\left(\mathrm{m}^{2}\right)$.

\section{Muscle Strength.}

The 1RM test was used to assess maximal strength on latissimus dorsi pulldown, leg extension, chest press, and leg curl to prevent fatigue from two consecutive upper- or lower-body RE. All participants were asked to warm up on an upright bike for five minutes at self-selected pace and performed 5-10 repetitions at 50\% of their bodyweights for a warm-up. Following the warm-up, participants performed the aforementioned resistance exercises at $50 \%$ of estimated 1RM followed by 3-5 attempts with two minutes rest between attempts and exercises. The highest weight lifted over the first and second visits was used to prescribe the exercise for the upper- and lower-body RE with or without BFR.

\section{Arterial Occlusion Pressure.}

The 13-cm wide nylon cuff (SC12D, Hokanson, Bellevue, WA, USA) was wrapped at the proximal end of the right arm or right leg. The ultrasound Doppler probe (GE Logiq 7, GE Healthcare, Milwaukee, WI) was placed on the brachial artery or femoral artery to detect the arterial blood flow. The cuff was inflated by a Rapid Cuff Inflation System (E20, Hokanson, Bellevue, WA, USA) to $50 \mathrm{mmHg}$ followed by $1 \mathrm{mmHg}$ increase per second until blood flow could not be detected. The pressures obtained during visit 1 and 2 were averaged and was recorded as AOP. 


\section{Exercise Protocol.}

The upper-body RE consisted of latissimus dorsi pulldown and chest press; the lower-body RE consisted of leg extension and leg curl. The RE with BFR consisted of 30-15-15-15 repetitions at $30 \%$ of 1 RM with 30 seconds and two minutes of rest between sets and exercises, respectively. The BFR was applied at $40 \%$ of $\mathrm{AOP}^{24}$ on the proximal end of both arms or legs using two 13cm nylon cuffs and a Rapid Cuff Inflation. The $40 \%$ of AOP was maintained during the RE and rest intervals between sets, and was released during the rest interval between exercises. The RE without BFR utilized four sets of eight repetitions at $70 \%$ of 1RM with 60 seconds and two minutes of rest between sets and exercises, respectively. The total exercise workloads of RE with BFR and RE without BFR were calculated as maximal strength $x 30 \%$ x 75 repetitions (1 set $\mathrm{x}$ 30 repetition +3 sets $\times 15$ repetitions) and maximal strength $\times 70 \% \times 32$ repetitions (4 sets $\times 8$ repetitions), respectively.

\section{Hemodynamics and Pulse Wave Reflections.}

All hemodynamics and pulse wave reflections were measured and calculated via a SphygmoCor XCEL (AtCor Medical, Sydney, Australia). After 10-minute rest in the supine position, the SphygmoCor XCEL was used to measure brachial BP twice, with each measurement separated by 1 minute. If the $2 \mathrm{BP}$ measurements were different over $5 \mathrm{mmHg}$, a third BP measurement was conducted. From there, we averaged the $2 \mathrm{BP}$ measurements that were within $5 \mathrm{mmHg}$. Pulse wave reflections were measured and calculated automatically after BP measurements. The RPP was calculated from HR multiplied by BSBP then divided by $100 \%$, and it is an indicative of myocardial oxygen demand and can be used to prescribe exercise for individuals with coronary artery disease. ${ }^{25}$ The BP waveforms consisted of a central forward wave (P1) and a peripheral backward wave $(\mathrm{P} 2)$. The central forward wave is generated by the ejection of stroke volume from the left ventricle, and when the central forward wave reaches peripheral vessels it is reflected and travels backward to left ventricle. ${ }^{23}$ The AP was calculated as the difference between P1 and P2 while the AIx was calculated as the AP divided by PP then multiplied by $100 \%$. Since AIx is affected by $\mathrm{HR}^{25}$, AIx was normalized at a HR of $75 \mathrm{bpm}$ by the computer. The AIx and AIx@75 are widely used determinants of wave reflection and risk factors of cardiovascular diseases. ${ }^{21}$ The Tr was obtained from the BP waveform and is defined as the time 
that the central forward wave travels to the peripheral arterioles and travels back to the aorta, this is also an indicative of arterial stiffness. ${ }^{23}$ As artery becomes stiffer, the travel speed of central forward wave increases as the travel time between aorta and peripheral arterioles decreases. ${ }^{23}$ The $\Delta \mathrm{E}_{\mathrm{w}}$ was used to measure additional myocardial workload, oxygen demand, and perfusion on the left ventricle, and was calculated as: $1.333 \mathrm{x} \mathrm{AP} \mathrm{x} \mathrm{(ventricular} \mathrm{ejection} \mathrm{duration}-\mathrm{Tr}$ ) $\mathrm{x}$ $\pi / 4$, as 1.333 converts $\mathrm{mmHg} / \mathrm{s}$ to dyn $\bullet / \mathrm{cm}^{2}{ }^{27}$ The SEVR was calculated from the ratio of the area under the curve of diastolic pressure by the area under the curve of systolic pressure and is the valid measure between oxygen supply and demand, which indicates myocardial perfusion and microvascular function. ${ }^{22}$

\section{Statistical analysis.}

Based on the AIx@75 $\left(\eta_{\mathrm{p}}{ }^{2}=0.5\right)$ from previous data ${ }^{4}, 22$ participants were needed to maintain an alpha of 0.05 and power of 0.8 . Normality of all data were analyzed with a KolmogorovSmirnoff test. All variables were classified as normally distributed thus a 2-way analysis of variance (ANOVA) was used to determine if there are any significant differences at rest between groups (upper- and lower-body) and conditions (BFR and without BFR). A 2x2x5 repeated measures ANOVA was used to test the effects of resistance exercise groups across conditions and repeated factor of time on hemodynamics [HR, BSBP, BDBP, RPP] and pulse wave reflections [aortic systolic BP (ASBP), aortic diastolic BP (ADBP), AP, AIx, AIx@75, Tr, $\Delta \mathrm{E}_{\mathrm{w}}$, and SEVR]. If there were significant interactions, paired t-tests were used to determine significance using Bonferroni correction factor for repeated measures. Mauchly's test was used to examine sphericity for all variables. If Mauchly's test was significant, the results of Greenhouse-Geisser correction were reported. Significant level was set at $\mathrm{p} \leq 0.05$. IBM SPSS 25.0 (IBM, Armonk, NY, USA) was used for all statistical analyses. All data are presented as mean \pm standard deviation $(\mathrm{SD})$.

\section{RESULTS}

Participants characteristics and 1RMs on 4 different exercises are presented in Table 1. Hemodynamics are presented in Table 2. There was no difference in total exercise workloads between upper- and lower-body with and without BFR $(\mathrm{p}=0.08)$. There was a significant threeway interaction in $\operatorname{RPP}\left(\mathrm{F}_{(4,88)}=3.07, \mathrm{p}=0.02\right)$ such that upper-body $\mathrm{RE}$ with $\mathrm{BFR}$ had 
significantly lower RPP compared to upper-body RE without BFR, lower-body RE with and without BFR at 10 minutes post-exercise, and upper-body RE without BFR, lower-body RE with BFR at 25 minutes post-exercise. There was a main effect of time in $\mathrm{HR}\left(\mathrm{F}_{(2.12,46.55)}=66.8\right.$, $\mathrm{p} \leq 0.001$ ) such that upper- and lower-body RE with or without BFR increased HR at 10, 25, 40, and 55 minutes post-exercise compared to Rest, 10 compared to 25 , 40, and 55 minutes postexercise, 25 compared to 40 and 55 minutes post-exercise. There were significant group-by-time interactions for $\operatorname{BSBP}\left(\mathrm{F}_{(4,88)}=13.2, \mathrm{p} \leq 0.001\right)$, and $\operatorname{BDBP}\left(\mathrm{F}_{(4,88)}=18.4, \mathrm{p} \leq 0.001\right)$. Lower-body $R E$ with or without BFR induced significantly higher BSBP compared to Rest and upper-body RE with or without BFR at 10 minutes post-exercise. For BDBP, upper-body RE with or without BFR significantly lowered BDBP compared to Rest and lower-body RE with or without BFR at 10 minutes post-exercise.

Pulse wave reflections are presented at Table 3 . There were significant group by time interactions in $\operatorname{ASBP}\left(\mathrm{F}_{(2.89,63.66)}=7.93, \mathrm{p}<0.001\right), \operatorname{ADBP}\left(\mathrm{F}_{(4,88)}=21.63, \mathrm{p}<0.001\right)$, and $\Delta \mathrm{E}_{\mathrm{w}}$ $\left(\mathrm{F}_{(2.07,45.49)}=9.76, \mathrm{p}<0.001\right)$. There was no change in ASBP and ADBP after upper-body RE with BFR and after lower-body RE without BFR, respectively. However, lower-body RE with BFR significantly increased ASBP at 10 and 25 minutes post-exercise compared to Rest and upperbody RE with BFR while lower-body RE without BFR increased ASBP at 10 minutes postexercise compared to Rest and upper-body RE without BFR. Upper-body RE with or without BFR significantly lowered ADBP at 10 minutes post-exercise compared to Rest and lower-body $\mathrm{RE}$ with or without BFR. There were significant 3-way interactions for $\mathrm{AP}\left(\mathrm{F}_{(4,88)}=3.66\right.$, $\mathrm{p}=0.008)$, AIx $\left(\mathrm{F}_{(4,88)}=3,54, \mathrm{p}=0.01\right)$, and AIx@ $75\left(\mathrm{~F}_{(4,88)}=2.83, \mathrm{p}=0.029\right)$ (Figure 1). The AP was elevated after upper-body RE with or without BFR at 10 and 25 minutes post-exercise compared to lower-body RE with or without BFR; the AIx and AIx @75 were augmented after upper-body $\mathrm{RE}$ with or without BFR at 10 minutes post-exercise compared to lower-body RE with or without BFR, and after upper-body RE without BFR compared to lower-body RE without BFR at 25 minutes post-exercise. Upper-body RE without BFR also induced higher AIx @ 75 at 25 minutes post-exercise compared to upper-body RE with BFR. There was significantly group by time interaction $\left(\mathrm{F}_{(2.07,45.49)}=9.76, \mathrm{p}<0.001\right)$ for $\Delta \mathrm{E}_{\mathrm{w}}$ such that it significantly increased after upper-body RE with or without BFR at 10 minutes post-exercise compared to Rest and lowerbody RE with or without BFR. There was main effect of time $\left(\mathrm{F}_{(4,84)}=2.81, \mathrm{p}=0.03\right)$ for Tr such that lower-body RE with or without BFR significantly reduced at 10 minutes post-exercise 
compared to Rest; there was also main effect of group $\left(\mathrm{F}_{(1,21)}=11.88, \mathrm{p}=0.002\right)$ for $\operatorname{Tr}$ such that lower-body RE with BFR significantly reduced at 10, 25, and 40 minutes post-exercise compared upper-body RE with BFR. There was main effect of time in $\operatorname{SEVR}\left(\mathrm{F}_{(2.22,48.80)}=82.6\right.$, $\mathrm{p} \leq 0.001)$ such that upper- or lower-body RE with or without BFR increased SEVR at 10, 25, 40, and 55 minutes post-exercise compared to Rest, 10 compared to 25, 40, and 55 minutes postexercise, 25 compared to 40 and 55 minutes post-exercise.

\section{DISCUSSION}

To our knowledge, this is the first study to evaluate the difference between upper- and lowerbody RE with and without BFR on hemodynamics and pulse wave reflections in young resistance-trained individuals. The novel findings of the present study are that a) All upper- and lower-body RE with and without BFR significantly increased RPP up to 60 minutes postexercise, however, upper-body RE with BFR at 10 minutes post-exercise produced a significantly attenuated RPP compared to the other group and condition, b) either upper- or lower-body RE with or without BFR significantly increased HR up to 60 minutes post-exercise, c) upper-body RE with or without BFR significantly decreased BDBP and ADBP while lowerbody RE with or without BFR significantly increased BSBP and ASBP, d) upper-body RE with or without BFR significantly elevated AP, AIx, AIx@75, $\triangle \mathrm{E}_{\mathrm{w}}$ and decreased SEVR while lowerbody RE with or with BFR significantly augmented AIx@75 and reduced Tr up to 10 minutes post-exercise. Collectively, these data show that upper- or lower-body RE has a profound effect on the cardiovascular parameters. Regardless of upper- or lower-body RE, there was no difference between with and without BFR on cardiovascular parameters.

In agreement with our hypothesis and previous studies, HR was significantly elevated after upper-body RE with ${ }^{4}$ or without ${ }^{4,6-8,28}$ BFR. In addition, our findings and previous studies demonstrate that upper-body RE with ${ }^{4,9}$ or without ${ }^{4,10,28}$ BFR does not alter BSBP. However, in contrast to our hypothesis and previous findings ${ }^{4,9,10}$, upper-body RE with or without BFR significantly reduced BDBP with no difference between upper-body RE with and without BFR.

Maior et al. (2015) reported that upper-body RE with BFR significantly reduced BSBP and BDBP compared to upper-body RE without BFR, which suggested that the length of postexercise hypotension might result from not only rest intervals and intensity, but also BFRinduced ischemia. This suggests that RE with BFR may be an effective stimulus to promote post- 
exercise hypotension compared to RE without BFR. ${ }^{28}$ Machado et al. (2020) reported that RPP significantly increased at five minutes and returned to resting levels at 15 minutes after upperbody RE without BFR while we observed an increased RPP up to 55 minutes after upper-body RE with and without BFR. The difference may result from different exercise volumes. ${ }^{30}$ Machado et al. (2020) performed bench press only for 2 sets of $10 \mathrm{RM}$ or $20 \mathrm{RM}$ while we performed latissimus dorsi pulldown and chest press for four sets each. Our findings suggest that the changes in BSBP and BDBP between upper-body RE with and without BFR are not affected by different rest intervals, intensity, or ischemia. Surprisingly, the changes in RPP were different between upper-body RE with and without BFR at 10 and 25 minutes post-exercise. Although both conditions had similar HR and BSBP, upper-body RE with BFR had slightly lower HR and BSBP, which results in significant lower RPP. This is important to note as it implies less myocardial work when performing upper-body RE with BFR, which is a novel finding and offers interesting insight into myocardial oxygen demand and upper-body RE with BFR. Researchers have demonstrated that HR increases significantly to compensate for a significantly reduced stroke volume in order to maintain cardiac output during RE with or without BFR. ${ }^{31,32}$ However, both of these studies ${ }^{31,32}$ performed lower-body RE with and without BFR. On the other hand, Brandner et al. (2015) reported no changes in HR, stroke volume, and cardiac output after biceps curls with and without BFR, however, these biceps curls were unilateral. Although there were no significant differences in HR and BSBP between upper-body RE with and without BFR in the present study, the slightly lower HR in this condition multiplied by non-significantly lower BSBP (-3mmHg from Rest) resulted in a significantly lower RPP after upper-body RE with BFR. This suggests that upper-body RE with BFR may require less myocardial oxygen demand compared to upper-body RE without BFR. It is important to note that the increases in AP, AIx, AIx@75, and $\Delta \mathrm{E}_{\mathrm{w}}$ might increase arterial stiffness while the decrease in SEVR indicated insufficient coronary flow, vasodilation, and microvascular function after acute upper-body RE with and without BFR in the present study.

In agreement with our hypothesis and previous studies, lower-body RE with ${ }^{12,13}$ or without ${ }^{13-16,28}$ significantly increased HR. In contrast to our hypothesis, the present study showed that lower-body RE with or without BFR significantly increased in BSBP at 10 minutes postexercise and returned to resting levels at 25 minutes post-exercise. Most studies have shown no change in BSBP 10 minutes or longer after lower-body RE with ${ }^{11,12,14}$ or without BFR. $^{11,14,16,28}$ 
However, in agreement with our hypothesis, and previous studies, there appears to be no change in BDBP after lower-body RE with ${ }^{11,12,14}$ or without BFR. ${ }^{11,14-16,28}$ Nevertheless, studies reported that RPP significantly increased at five minutes and returned to resting levels at 15 minutes after lower-body RE with ${ }^{12}$ or without $\mathrm{BFR}^{30}$ while we observed an increased RPP up to 55 minutes after lower-body RE with and without BFR. Again, Rossow et al. (2012) performed knee extension with BFR for four sets of 30,15, 15, and 15 repetitions at 20\% 1RM and Machado et al. (2020) performed leg press without BFR for two sets of 10 RM or 20 RM while we performed leg extension and leg curl for four sets each. Therefore, it has been reported that resistance exercise, as well as BFR, stimulate the exercise pressor reflex, group III and IV muscle afferents, as known as mechanical reflex (compression of blood vessels) and metabolic reflex (accumulation of metabolites) which leads to increases in BSBP and BSDP. ${ }^{33,34}$ Therefore, it is difficult to compare changes in BP across numerous studies because the magnitude of changes in BP is mediated by different exercise intensities, volume, rest intervals, and pressure of cuffs.

Upper-body RE with ${ }^{4}$ or without ${ }^{4}{ }^{10} \mathrm{BFR}$, as well as the present study, suggest no change in ASBP. In contrast to our hypothesis and previous studies ${ }^{4,10}$ upper-body RE with or without BFR resulted in significantly decreased ADBP. Lefferts et al. (2014) demonstrated that upperbody RE without BFR significantly increased ASBP and decreased ADBP. Tomschi et al. (2018) demonstrated that ADBP was significantly reduced at 10 minutes while no change was reported for ASBP after upper-body RE without BFR. However, the present study demonstrated significantly decreased ADBP with no change on ASBP after upper-body RE with or without BFR. The different responses may be from different exercise intensity, number of exercises, or the time in which measurements were taken.

Rossow et al, (2012) and Figueroa and Vicil (2011) reported significantly augmented ASBP and ADBP immediately after lower-body RE with or without BFR that returned to resting levels at five ${ }^{12}$ or 30 minutes ${ }^{14}$ during recovery. However, Tomschi et al. (2018) did not find any changes in ASBP or ADBP after lower-body RE without BFR. Therefore, we did not measure ASBP or ADBP immediately after lower-body RE with or without BFR, but our results are different, the ASBP was still elevated at 10 minutes post-exercise.

In agreement with our hypothesis, a previous study ${ }^{4}$ demonstrated that upper-body RE with or without BFR significantly increased AP, AIx, and AIx@75 with no change in Tr. Similar 
studies have reported that upper-body RE without BFR significantly increased $\mathrm{AP}^{6}, \mathrm{AIx}^{6,8}$, and AIx@ $95^{6-8}$ with no change in $\operatorname{Tr}^{6}$. It is suggested that upper-body RE without BFR results in a shorter transit time of the backward traveling BP waveform from peripheral sites during the contractile phase might lead to increases in AP and AIx. ${ }^{10}$ In agreement with our hypothesis, the $\Delta \mathrm{E}_{\mathrm{w}}$ significantly increased after upper-body $\mathrm{RE}$ with or without $\mathrm{BFR}$, a previous study have reported the upper-body $\mathrm{RE}$ with BFR significantly elevated $\Delta \mathrm{E}_{\mathrm{w}}$ while upper-body $\mathrm{RE}$ without BFR did not show significant change. ${ }^{4}$ In addition, Tai et al. (2019) reported that SEVR was significantly decreased at 10 minutes after upper-body RE with or without BER which agree on the present finding. Tagawa et al. (2018) reported that 4-week upper-body RE without BFR significantly lower SEVR and there is a positive correlation between SEVR and central aortic compliance. Although we performed an acute bout of RE while Tagawa et al. (2018) conducted a long-term RE, the decrease in SEVR after upper-body RE with or without BFR might resulted from an increase in the area under the curve of systolic pressure. ${ }^{35}$

Unlike upper-body RE with or without BFR, lower-body RE with or without BFR did not change most pulse wave reflections (AP, AIx, and $\Delta \mathrm{E}_{\mathrm{w}}$ ) in the present study. There were significant differences on AIx@75 and Tr such that AIx@75 was elevated and Tr was reduced at 10 minutes after lower-body RE with or without BFR compared to Rest. Previous studies showed no change in AP or AIx five minutes after lower-body RE with ${ }^{12}$ or without BFR. ${ }^{36}$ However, Figueroa and Vicil (2011) reported decreases in AP and AIx 30 minutes after lowerbody RE with or without BFR. This response may have been mediated by post-exercise peripheral vasodilation. ${ }^{37}$ Despite the reported negative relationship between AIx and HR, AIx fluctuates by the magnitude of $\mathrm{BP}$ waveforms and $\mathrm{Tr}^{26}$ However, we did not observe the negative relationship between AIx and HR, nor changes in AP, but observed significantly reduced on Tr after lower-body RE with or without BFR in the present study. Therefore, the reduced Tr did not assist in mediating the significant changes in AIx in the present study. To our knowledge, no study has examined the effect of lower-body RE with or without BFR in $\triangle \mathrm{E}_{\mathrm{w}}$ and SEVR, we only can compare findings to whole-body RE without BFR. Parks et al. (2020) and Kingsley et al. (2017) reported no changes in $\Delta \mathrm{E}_{\mathrm{w}}$ and a significant reduction in SEVR 10 minutes after whole-body RE without $\mathrm{BFR}^{38,39}$ while Tai et al. (2018) showed there was a significant increase in $\Delta \mathrm{E}_{\mathrm{w}} 10$ minutes after whole-body $\mathrm{RE}$ without $\mathrm{BFR}{ }^{3}$ 
Several limitations in the present study should be noted. Firstly, we only recruited young, healthy individuals in the present study. The results should not be directly generalized to middleaged or older population or individuals with cardiovascular or metabolic diseases. Secondly, we used 2 different loads (30\% vs. 70\% 1RM) in the present study, which might mediate different responses in hemodynamics. However, the primary aim of the present study was to compare currently prevalent low-load RE with BFR to traditional high-load RE while keeping the total exercise workloads similar. Thirdly, sexes differences exist in hemodynamics and pulse wave reflections ${ }^{40}$, however, we did not have an equal number of men and women. Our original second aim was to recruit equal numbers of men and women so that we could provide general information without sex bias; however, it was difficult to schedule women due to the timing of their menstrual cycle. Therefore, the decision to include nine women in the present study was to meet our apriori power calculation, which indicated 22 participants were needed for the present study. And lastly, our health history questionnaire was not sensitive enough to determine if participants played in any collegiate or recreational sports, specific physical activity in which they were involved, or the total resistance training volume they performed per week.

\section{CONCLUSION}

This is the first study to investigate the differences between upper- and lower-body RE with and without BFR on hemodynamics and pulse wave reflections in young resistance-trained individuals. It is surprising that lower-body RE promoted a greater increase in BSBP and ASBP while upper-body RE induced a significant decrease in SDBP and ADBP which was contrary to our hypothesis. On the other hand, upper-body RE produced greater elevations in AP, AIx, AIx@75, and $\Delta \mathrm{E}_{\mathrm{w}}$ except Tr and SEVR which met our hypothesis. In addition, the cardiovasuclar responses between BFR and without BFR are similar regardless of upper- or lower-body RE. To conclude, upper-body RE generate greater changes on pulse wave reflections while lower-body RE induce greater elevations in systolic BP. In other words, lower-body RE produced a reduced impact on measures of pulse wave reflection compared to upper-body RE, and might have advantageous effects on cardiovascular function compared to upper-body RE. However, we only evaluated acute responses, future studies should exam chronic responses and adapations between upper- and lower-body RE on hemodynamics and measures of pulse wave reflection. 


\section{Disclosure statement}

No potential conflict of interest was reported by the authors.

\section{Reference}

1. Medicine ACoS. Progression models in resistance training for healthy adults. Medicine \& Science in Sports \& Exercise. 2009;41(3):687-708. doi:10.1249/MSS.0b013e3181915670

2. Lixandrão ME, Ugrinowitsch C, Berton R, et al. Magnitude of muscle strength and mass adaptations between high-load resistance training versus low-load resistance training associated with blood-flow restriction: a systematic review and meta-analysis. Sports Medicine. February 01 2018;48(2):361-378. doi:10.1007/s40279-017-0795-y

3. Tai YL, Gerhart H, Mayo X, Kingsley JD. Acute resistance exercise using free weights on aortic wave reflection characteristics. Clinical Physiology and Functional Imaging. 2018;38(1):145-150.

doi:10.1111/cpf.12396

4. Tai YL, Marshall EM, Glasgow A, Parks JC, Sensibello L, Kingsley JD. Pulse wave reflection responses to bench press with and without practical blood flow restriction. Applied Physiology, Nutrition, and Metabolism. 2019/04/01 2019;44(4):341-347, doi:10.1139/apnm-2018-0265

5. Kengne AP, Czernichow S, Huxley R, et al. Blood pressure variables and cardiovascular risk: new findings from ADVANCE. Hypertension (Dallas, Tex:1979). Aug 2009;54(2):399-404.

doi:10.1161/hypertensionaha.109.133041

6 . Augustine JA, Nunemacher KN, Heffernan KS. Menstrual phase and the vascular response to acute resistance exercise. journal article. European Journal of Applied Physiology. May 01 2018;118(5):937-946. doi:10.1007/s00421-018-3815-1

7. Lefferts W, Augustine J, Heffernan K. Effect of acute resistance exercise on carotid artery stiffness and cerebral blood flow pulsatility, Original Research. Frontier in Physiology. 2014-March-19

2014;5(101)doi:10,3389/fphys.2014.00101

8. Fahs CA, Heffernan KS, Fernhall B. Hemodynamic and vascular response to resistance exercise with Larginine. Medicine and science in sports and exercise. Apr 2009;41(4):773-9.

doi:10.1249/MSS.0b013e3181909d9d

9. Brandner CR, Kidgell DJ, Warmington SA. Unilateral bicep curl hemodynamics: Low-pressure continuous vs high-pressure intermittent blood flow restriction. Scandinavian Journal of Science \& Medicine in Sports. 2015;25(6):770-777. doi:10.1111/sms.12297

10. Okamoto T, Min S, Sakamaki-Sunaga M. Arterial compliance and stiffness following low-intensity resistance exercise. journal article. European Journal of Applied Physiology. February 01 2014;114(2):235-241. doi:10.1007/s00421-013-2770-0

11. Poton R, Polito MD. Hemodynamic response to resistance exercise with and without blood flow restriction in healthy subjects. Clinical Physiology and Functional Imaging. 2016;36(3):231-236.

doi:10.1111/cpf.12218 
12. Rossow LM, Fahs CA, Loenneke JP, et al. Cardiovascular and perceptual responses to blood-flowrestricted resistance exercise with differing restrictive cuffs. Clinical Physiology and Functional Imaging. 2012;32(5):331-337. doi:10.1111/j.1475-097X.2012.01131.x

13. Rossow LM, Fahs CA, Sherk VD, Seo D-i, Bemben DA, Bemben MG. The effect of acute blood-flowrestricted resistance exercise on postexercise blood pressure. Clinical Physiology and Functional Imaging. 2011;31(6):429-434. doi:10.1111/j.1475-097X.2011.01038.x

14. Figueroa A, Vicil F. Post-exercise aortic hemodynamic responses to low-intensity resistance exercise with and without vascular occlusion. Scandinavian Journal of Science \& Medicine in Sports.

2011;21(3):431-436. doi:10.1111/j.1600-0838.2009.01061.x

15. Heffernan KS, Jae SY, Edwards DG, Kelly EE, Fernhall B. Arterial stiffness following repeated Valsalva maneuvers and resistance exercise in young men. Applied physiology, nutrition, and metabolism $=$ Physiologie appliquee, nutrition et metabolisme. Apr 2007;32(2):257-64. doi:10.1139/h06-107

16. Heffernan KS, Rossow L, Jae SY, Shokunbi HG, Gibson EM, Fernhall B. Effect of single-leg resistance exercise on regional arterial stiffness. European Journal of Applied Physiology. 2006/09/01

2006;98(2):185-190. doi:10.1007/s00421-006-0259-9

17. London GM, Blacher J, Pannier B, Guérin AP, Marchais SJ, Safar ME. Arterial wave reflections and survival in end-stage renal failure. Hypertension (Dallas, Tex : 1979), Sep 2001;38(3):434-8.

doi:10.1161/01.hyp.38.3.434

18. Weber T, Auer J, O'Rourke Michael F, et al. Arterial stiffness, wave reflections, and the risk of coronary artery disease. Circulation. 2004/01/20 2004;109(2):184-189.

doi:10.1161/01.CIR.0000105767.94169.E3

19. O'Rourke MF. Wave travel and reflection in the arterial system. Journal of hypertension Supplement : official journal of the International Society of Hypertension. Dec 1999;17(5):S45-7.

20. O'Rourke MF, Adji A. An updated clinical primen on large artery mechanics: implications of pulse waveform analysis and arterial tonometry. Current opinion in cardiology. Jul 2005;20(4):275-81. doi:10.1097/01.hco.0000166595.44711.6f

21. Hashimoto J, Nichols WW, O'Rourke MF, Imai Y. Association between wasted pressure effort and left ventricular hypertrophy in hypertension: influence of arterial wave reflection. American Journal of Hypertension. 2008;21(3):329-333. doi:10.1038/ajh.2007.49

22. Tsiachris D, Tsioufis $C$, Syrseloudis $D$, et al. Subendocardial viability ratio as an index of impaired coronary flow reserve in hypertensives without significant coronary artery stenoses. Journal of human hypertension. Jan 2012;26(1):64-70. doi:10.1038/jhh.2010.127

23. Nichols WW, Singh BM. Augmentation index as a measure of peripheral vascular disease state. Current opinion in cardiology. Sep 2002;17(5):543-51. doi:10.1097/00001573-200209000-00016

24. Loenneke JP, Kim D, Fahs CA, et al. The influence of exercise load with and without different levels of blood flow restriction on acute changes in muscle thickness and lactate. Clinical Physiology and Functional Imaging. 2017;37(6):734-740. doi:10.1111/cpf.12367

25. Wilkinson IB, MacCallum H, Flint L, Cockcroft JR, Newby DE, Webb DJ. The influence of heart rate on augmentation index and central arterial pressure in humans. The Journal of Physiology. 2000/05/01 2000;525(1):263-270. doi:10.1111/j.1469-7793.2000.t01-1-00263.x

26. Casey DP, Curry TB, Joyner MJ, Charkoudian N, Hart EC. Relationship between muscle sympathetic nerve activity and aortic wave reflection characteristics in young men and women. Hypertension (Dallas, Tex : 1979). Mar 2011;57(3):421-7. doi:10.1161/hypertensionaha.110.164517

27. Tomschi F, Köster P, Predel H-G, Lay D, Bloch W, Grau M. Acute effects of lower and upper bodyresistance training on arterial stiffness, peripheral, and central blood pressure in young normotensive women. Sport Sciences for Health. 2018/08/01 2018;14(2):357-363. doi:10.1007/s11332-018-0440-7 
28. Maior AS, Simão R, Martins MSR, de Salles BF, Willardson JM. Influence of blood flow restriction during low-intensity resistance exercise on the postexercise hypotensive response. The Journal of Strength \& Conditioning Research. 2015;29(10)

29. Machado CLF, Bgeginski R, De Castro C, Wilhelm EN, Pinto RS. Acute Hemodynamic responses to repetitions to failure using different resistance exercises and protocols in normotensive men: a crossover study. Clinical and experimental hypertension (New York, NY : 1993). Jul 3 2020;42(5):401-408. doi:10.1080/10641963.2019.1676772

30. May AK, Brandner CR, Warmington SA. Hemodynamic responses are reduced with aerobic compared with resistance blood flow restriction exercise. Physiological reports. Feb 2017;5(3)doi:10.14814/phy2.13142

31. Pinto RR, Karabulut M, Poton R, Polito MD. Acute resistance exercise with blood flow restriction in elderly hypertensive women: haemodynamic, rating of perceived exertion and blood lactate. Clin Physiol Funct Imaging. Jan 2018;38(1):17-24. doi:10.1111/cpf.12376

32. Alam M, Smirk FH. Observations in man upon a blood pressure raising reflex arising from the voluntary muscles. The Journal of Physiology. 1937/06/03 1937;89(4):372-383.

doi:10.1113/jphysiol.1937.sp003485

33. Alam M, Smirk FH. Observations in man on a pulse-accelerating reflex from the voluntary muscles of the legs. The Journal of Physiology. 1938/03/14 1938;92(2):167-177.

doi:10.1113/jphysiol.1938.sp003592

34. Tagawa K, Choi Y, Ra SG, Yoshikawa T, Kumagai H, Maeda S. Resistance training-induced decrease in central arterial compliance is associated with decreased subendocardial viability ratio in healthy young men. Applied physiology, nutrition, and metabolism = Physiologie appliquee, nutrition et metabolisme. May 2018;43(5):510-516. doi:10.1139/apnm-2017-0449

35. Fryer S, Stone K, Dickson T, et al. Reliability of oscillometric central blood pressure responses to lower limb resistance exercise. Atherosclerosis. 2018/01/01/ 2018;268:157-162.

doi:https://doi.org/10.1016/j.atherosclerosis.2017.11.031

36. Munir S, Jiang B, Guilcher A, et al. Exercise reduces arterial pressure augmentation through vasodilation of muscular arteries in humans. American journal of physiology Heart and circulatory physiology. Apr 2008;294(4):H1645-50. doi:10.1152/ajpheart.01171.2007

37. Kingsley JD, Tai YL, Mayo X, Glasgow A, Marshall E. Free-weight resistance exercise on pulse wave reflection and arterial stiffness between sexes in young, resistance-trained adults. European journal of sport science. Sep 2017,17(8):1056-1064. doi:10.1080/17461391.2017.1342275

38. Parks JC, Marshall EM, Tai YL, Kingsley JD. Free-weight versus weight machine resistance exercise on pulse wave reflection and aortic stiffness in resistance-trained individuals. European journal of sport science. Aug 2020;20(7):944-952. doi:10.1080/17461391.2019.1685007

39. Hayward CS, Kelly RP. Gender-related differences in the central arterial pressure waveform. Journal of the American College of Cardiology. Dec 1997;30(7):1863-71. doi:10.1016/s0735-1097(97)00378-1 40. Doonan RJ, Mutter A, Egiziano G, Gomez YH, Daskalopoulou SS. Differences in arterial stiffness at rest and after acute exercise between young men and women. Hypertension research : official journal of the Japanese Society of Hypertension. Mar 2013;36(3):226-31. doi:10.1038/hr.2012.158

Table 1. Participant characteristics $(\mathrm{N}=23)$

\begin{tabular}{|c|c|}
\hline & Participants \\
\hline
\end{tabular}




\begin{tabular}{|l|c|}
\hline Age $(\mathrm{yr})$ & $22 \pm 2$ \\
\hline Height $(\mathrm{m})$ & $1.72 \pm 0.09$ \\
\hline Weight $(\mathrm{kg})$ & $72.3 \pm 12.1$ \\
\hline Body Mass Index $\left(\mathrm{kg} / \mathrm{m}^{2}\right)$ & $24.3 \pm 2.9$ \\
\hline Year of training $(\mathrm{yr})$ & $6 \pm 3$ \\
\hline 1-repetition Maximum $(\mathrm{kg})$ & \\
\hline Latissimus Dorsi Pulldown & $127 \pm 34$ \\
\hline Chest Press & $79 \pm 30$ \\
\hline Knee Extension & $117 \pm 25$ \\
\hline Knee Flexion & $78 \pm 19$ \\
\hline
\end{tabular}

Data are presented as mean $\pm \mathrm{SD}$

Abbreviation: $\mathrm{BFR}=$ blood flow restriction; $\mathrm{RE}=$ resistance exercise.

Table 2. Hemodynamics ( $\mathrm{N}=23)$

\begin{tabular}{|c|c|c|c|c|c|}
\hline \multirow{2}{*}{ Variables } & \multirow{2}{*}{ Time } & \multicolumn{2}{|c|}{ Upper-Body } & \multicolumn{2}{|c|}{ Lower-Body } \\
\hline & & BFR & Without BFR & BFR & Without BFR \\
\hline \multirow{5}{*}{$\begin{array}{l}\text { Heart Rate } \\
\quad(\mathrm{bpm})\end{array}$} & Rest & $59 \pm 10$ & $58 \pm 10$ & $60 \pm 10$ & $59 \pm 9$ \\
\hline & 10 & $72 \pm 10 *$ & $76 \pm 13 *$ & $74 \pm 12 *$ & $74 \pm 13 *$ \\
\hline & 25 & $67 \pm 10 * \dagger$ & $71 \pm 12 * \dagger$ & $68 \pm 11 * \dagger$ & $69 \pm 11 * \dagger$ \\
\hline & 40 & $64 \pm 10 *+t$ & $66 \pm 12 *+t$ & $64 \pm 11 *+t$ & $64 \pm 11 *+t$ \\
\hline & 55 & $64 \pm 10 *+t$ & $64 \pm 14 *+\$$ & $64 \pm 11 *+t$ & $65 \pm 11 *+t$ \\
\hline \multirow{5}{*}{$\begin{array}{l}\text { Brachial } \\
\text { Systolic } \\
\text { Blood } \\
\text { Pressure } \\
(\mathrm{mmHg})\end{array}$} & Rest & $117 \pm 9$ & $117 \pm 11$ & $117 \pm 10$ & $117 \pm 11$ \\
\hline & 10 & $114 \pm 10 \S$ & $118 \pm 10 \S$ & $125 \pm 11 *$ & $124 \pm 12^{*}$ \\
\hline & 25 & $116 \pm 11 \S$ & $117 \pm 10$ & $122 \pm 13$ & $119 \pm 11 \dagger$ \\
\hline & 40 & $115 \pm 11$ & $118 \pm 12$ & $118 \pm 12 \dagger t$ & $118 \pm 13 \dagger$ \\
\hline & 55 & $117 \pm 11$ & $118 \pm 11$ & $119 \pm 12 \dagger$ & $120 \pm 13$ \\
\hline \multirow{5}{*}{$\begin{array}{c}\text { Brachial } \\
\text { Diastolic } \\
\text { Blood } \\
\text { Pressure } \\
(\mathrm{mmHg})\end{array}$} & Rest & $65 \pm 5$ & $65 \pm 6$ & $65 \pm 6$ & $64 \pm 6$ \\
\hline & 10 & $58 \pm 6 * \S$ & $60 \pm 5 * \S$ & $68 \pm 5$ & $67 \pm 5$ \\
\hline & 25 & $62 \pm 5 \uparrow \S$ & $62 \pm 6+\S$ & $67 \pm 6$ & $67 \pm 6$ \\
\hline & 40 & $63 \pm 6 \dagger$ & $61 \pm 5 \S$ & $65 \pm 5$ & $66 \pm 7$ \\
\hline & 55 & $64 \pm 6 \dagger$ & $64 \pm 5 \dagger$ & $66 \pm 5$ & $64 \pm 7$ \\
\hline
\end{tabular}

Data are presented as mean $\pm \mathrm{SD}$

Abbreviation: $\mathrm{BFR}=$ blood flow restriction.

${ }^{*} \mathrm{p}<0.05$, different from rest; $\uparrow \mathrm{p}<0.05$, different from $10 ; \ddagger \mathrm{p}<0.05$, different from 25 
$\S \mathrm{p}<0.05$, different from lower-body at the same condition and time

$\$ \mathrm{p}<0.05$, different from lower-body at different condition

$\# \mathrm{p}<0.05$, different from upper-body without BFR at the same group and time

Table 3. Pulse wave reflections $(\mathrm{N}=23)$

\begin{tabular}{|c|c|c|c|c|c|}
\hline \multirow{2}{*}{ Variables } & \multirow{2}{*}{ Time } & \multicolumn{2}{|c|}{ Upper-Body } & \multicolumn{2}{|c|}{ Lower-Body } \\
\hline & & BFR & Without BFR & (BFR) & Without BFR \\
\hline \multirow{5}{*}{$\begin{array}{c}\text { Aortic } \\
\text { Systolic } \\
\text { Blood } \\
\text { Pressure } \\
(\mathrm{mmHg})\end{array}$} & Rest & $103 \pm 6$ & $102 \pm 8$ & $102 \pm 8$ & $103 \pm 8$ \\
\hline & 10 & $101 \pm 9 \S$ & $104 \pm 7 \S$ & $108 \pm 9 *$ & $108 \pm 10 *$ \\
\hline & 25 & $101 \pm 8 \S$ & $103 \pm 8$ & $106 \pm 10^{*}$ & $104 \pm 9 \dagger$ \\
\hline & 40 & $100 \pm 9$ & $101 \pm 9$ & $102 \pm 9+t$ & $103 \pm 11 \dagger$ \\
\hline & 55 & $102 \pm 9$ & $103 \pm 9$ & $103 \pm 9$ & $103 \pm 10 \dagger$ \\
\hline \multirow{5}{*}{$\begin{array}{c}\text { Aortic } \\
\text { Diastolic } \\
\text { Blood } \\
\text { Pressure } \\
(\mathrm{mmHg})\end{array}$} & Rest & $65 \pm 5$ & $65 \pm 9$ & $66 \pm 6$ & $65 \pm 6$ \\
\hline & 10 & $59 \pm 6 * \S$ & $61 \pm 5 * \S$ & $69 \pm 5$ & $69 \pm 6$ \\
\hline & 25 & $63 \pm 6+\$$ & $63 \pm 6+\$$ & $69 \pm 6$ & $69 \pm 5$ \\
\hline & 40 & $64 \pm 6 \dagger$ & $63 \pm 5 \S$ & $66 \pm 6$ & $67 \pm 7$ \\
\hline & 55 & $66 \pm 6 \dagger$ & $66 \pm 5 \dagger$ & $67 \pm 5$ & $66 \pm 7$ \\
\hline \multirow{10}{*}{$\operatorname{Tr}(\mathrm{ms})$} & Rest & $148.8 \pm 3.9$ & $149.2 \pm 5.2$ & $148.0 \pm 4.6$ & $148.3 \pm 4.9$ \\
\hline & 10 & $149.9 \pm 8.7 \S$ & $148.3 \pm 5.6$ & $145.0 \pm 4.1^{*}$ & $145.6 \pm 5.9 *$ \\
\hline & 25 & $151.7 \pm 7.1 \S$ & $148.1 \pm 6.6$ & $146.5 \pm 3.7 \dagger$ & $147.2 \pm 6.2$ \\
\hline & & $151.3 \pm 6.2 \S$ & $151.7 \pm 11.4$ & $147.2 \pm 4.1+t$ & $148.3 \pm 5.6+t$ \\
\hline & 55 & $148.8 \pm 6.8 \$ \eta$ & $149.2 \pm 7.2$ & $148.5 \pm 4.4 \dagger \eta$ & $147.3 \pm 4.9$ \\
\hline & Rest & $1.53 \pm 0.31$ & $1.57 \pm 0.32$ & $1.49 \pm 0.29$ & $1.52 \pm 0.28$ \\
\hline & & $1.10 \pm 0.18 *$ & $1.04 \pm 0.23 *$ & $1.05 \pm 0.26 *$ & $1.09 \pm 0.26 *$ \\
\hline & 25 & $1.26 \pm 0.25 * \dagger$ & $1.17 \pm 0.25 * \dagger$ & $1.24 \pm 0.28 * \dagger$ & $1.25 \pm 0.29 * \dagger$ \\
\hline & 40 & $1.38 \pm 0.26 *+t$ & $1.32 \pm 0.29 *+t$ & $1.36 \pm 0.28 *+t$ & $1.37 \pm 0.29 *+4$ \\
\hline & 55 & $1.41 \pm 0.27 *+t$ & $1.43 \pm 0.40 *+t$ & $1.41 \pm 0.30 *+t$ & $1.38 \pm 0.29 *+t$ \\
\hline
\end{tabular}

Data are presented as mean $\pm \mathrm{SD}$

Abbreviation: $\mathrm{BFR}=$ blood flow restriction; $\mathrm{SEVR}=$ subendocardial viability ratio; $\mathrm{Tr}=\operatorname{transit}$ time of reflected wave.

${ }^{*} \mathrm{p}<0.05$, different from rest; $\uparrow \mathrm{p}<0.05$, different from $10 ; \$ \mathrm{p}<0.05$, different from 25 $\eta \mathrm{p}<0.05$, different from 40 
$\S p<0.05$, different from lower-body at the same condition and time $\# p<0.05$, different from upper-body without BFR at the same group and time

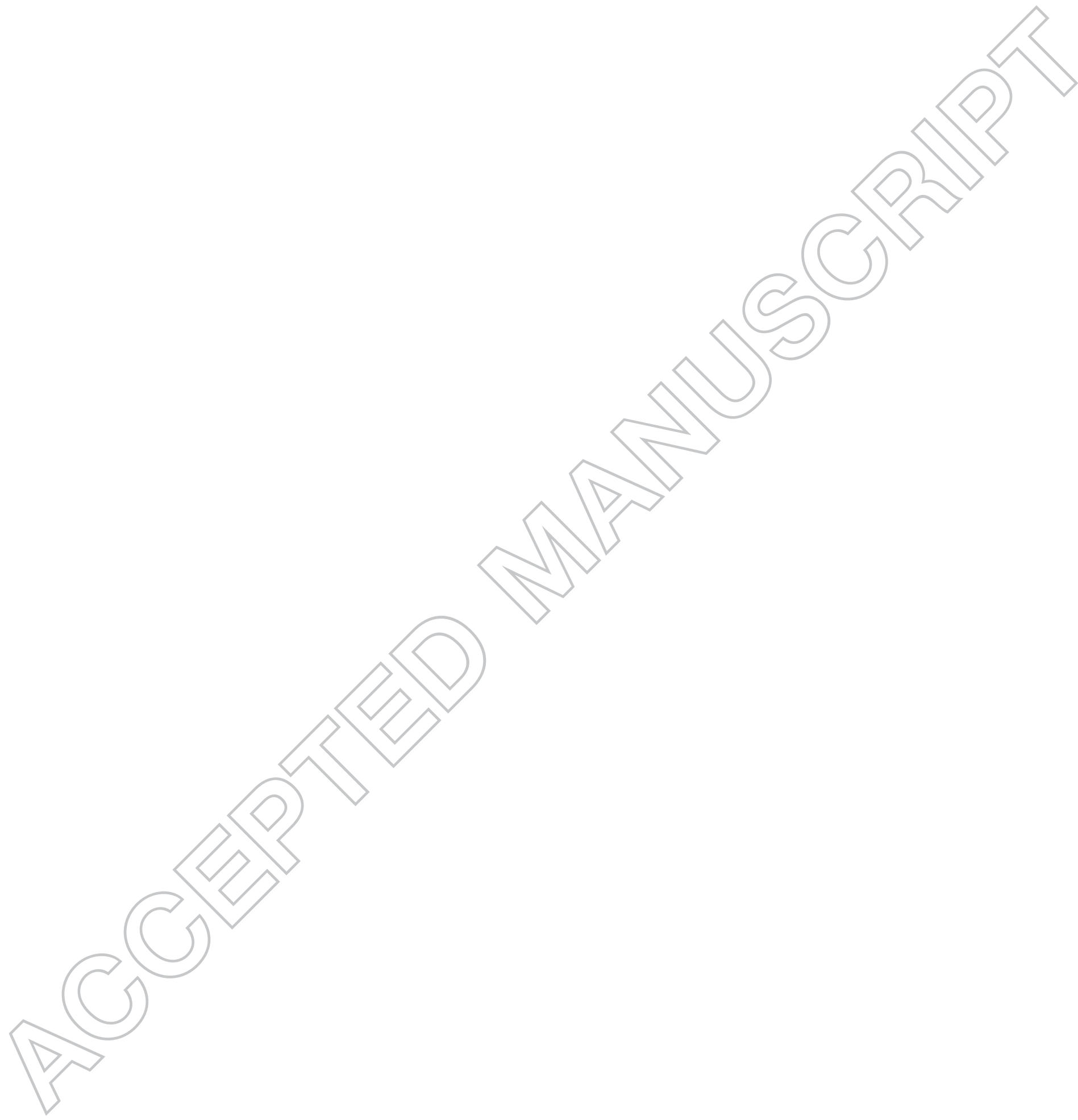




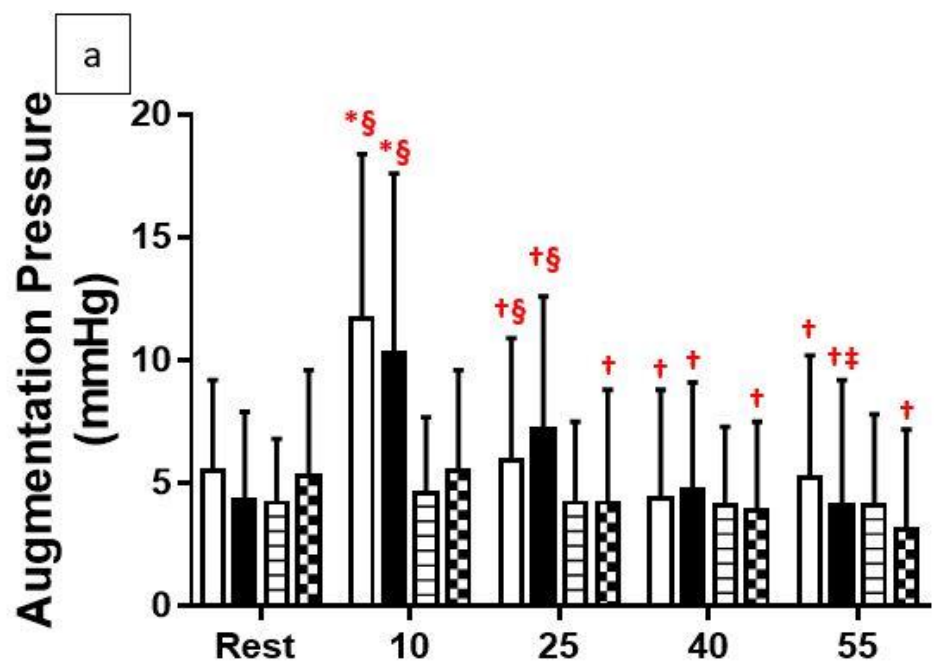

$\square$ Upper-Body RE with BFR

- Upper-Body RE without BFR

$\square$ Lower-Body RE with BFR

Wower-Body RE without BFR
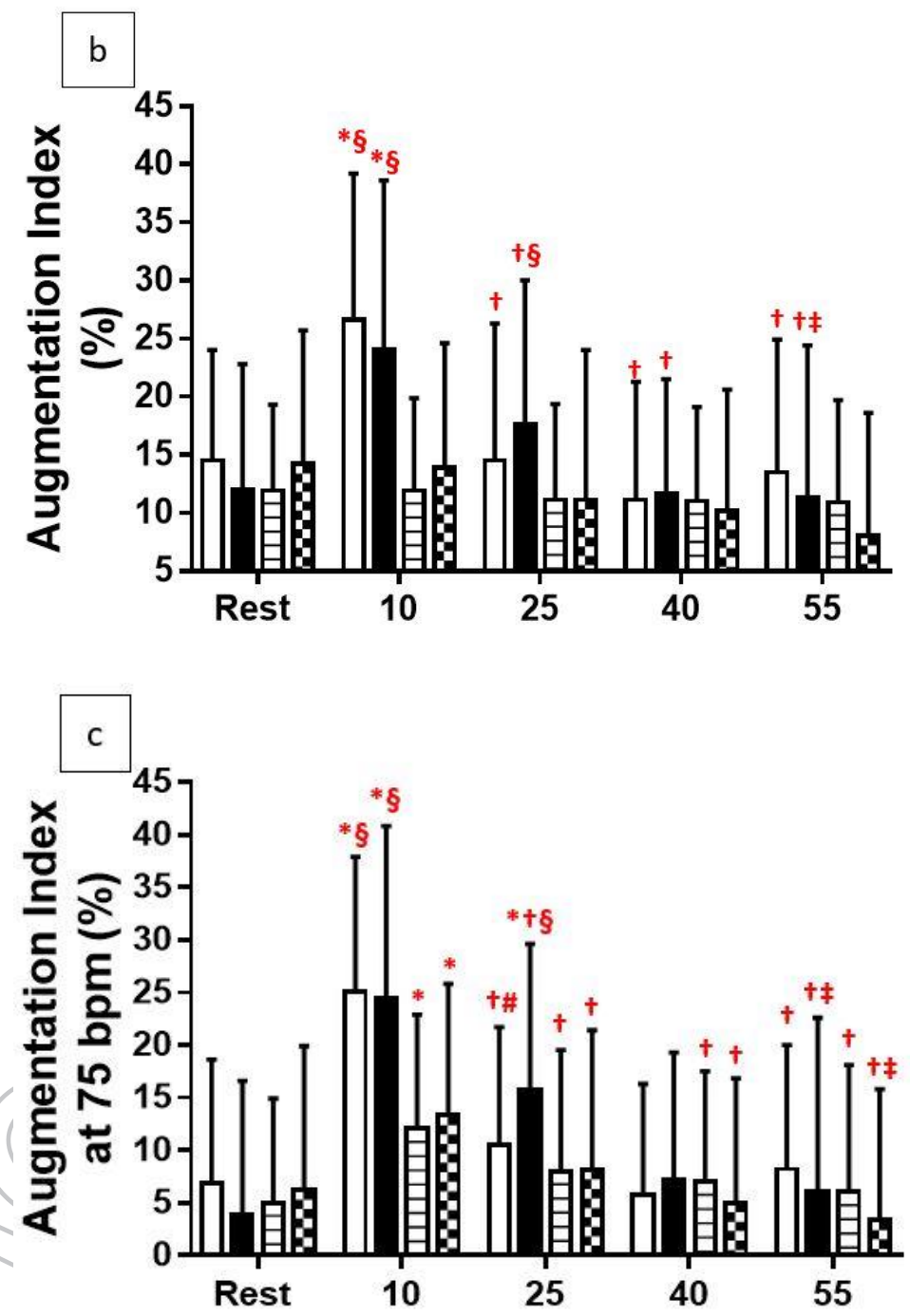

$\square$ Upper-Body RE with BFR

- Upper-Body RE without BFR

$\square$ Lower-Body RE with BFR

Wo Lower-Body RE without BFR $\square$ Upper-Body RE with BFR

- Upper-Body RE without BFR

$\boxminus$ Lower-Body RE with BFR

$\infty$ Lower-Body RE without BFR 


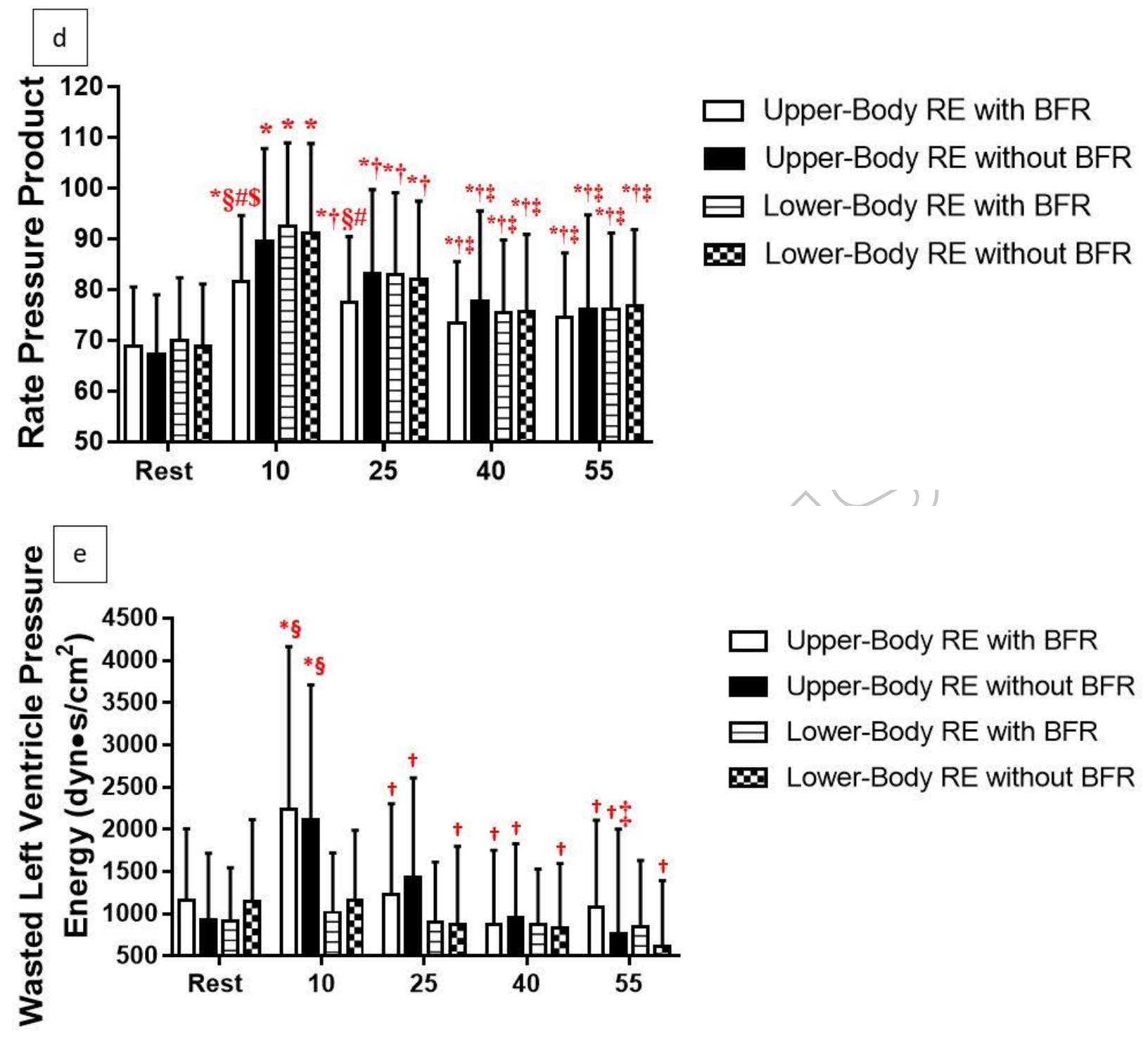

Figure 1. Absolute values in (a) augmentation pressure, (b) augmentation index, (c) augmentation index at $75 \mathrm{bpm}$ (d) rate pressure product, and (e) wasted left ventricle pressure energy at rest, 10, 25, 40, and 55 after upper- and lower-body resistance exercise with and without blood flow restriction in young resistance-trained individuals $(\mathrm{N}=23)$.

Data are presented as mean \pm SD

Abbreviation: $\mathrm{BFR}=$ blood flow restriction; $\mathrm{RE}=$ resistance exercise .

${ }^{*} \mathbf{p}<0.05$, different from rest; $\uparrow p<0.05$, different from $10 ; \$ p<0.05$, different from 25 $\S p<0.05$, different from lower-body at the same condition and time 
$\# p<0.05$, different from upper-body without BFR at the same group and time $\$ p<0.05$, different from lower-body at different condition 